\title{
DESENVOLVIMENTO DE UM SISTEMA DIDÁTICO REDUZIDO DE ENSAIO DE PERFIS DE PAREDE FINA A TORÇÃO PARA O ENSINO DE RESISTÊNCIA DOS MATERIAIS
}

\author{
André Campos de Moura - andre.moura@ uel.br \\ Gilberto Carbonari-carbonar@uel.br \\ Universidade Estadual de Londrina, Departamento de Estruturas, CTU \\ Rodovia Celso Garcia Cid, PR 445 Km 380, Campus Universitário, Cx. Postal 10.011 \\ CEP 86.057-970 - Londrina - PR
}

\author{
Antonio Roberto Fabri Leite - antonioroberto08@ hotmail.com \\ Universidade Estadual de Londrina, Curso de Engenharia Civil, CTU \\ Rodovia Celso Garcia Cid, PR 445 Km 380, Campus Universitário, Cx. Postal 10.011 \\ CEP 86.057-970 - Londrina - PR
}

\begin{abstract}
Resumo: No ensino de Resistência dos Materiais e Análise de Estruturas, uma das principais dificuldades encontrada por docentes é a dificuldade, por parte dos alunos, para entender conceitos básicos de uma forma mais prática, devido principalmente pelo elevado grau de abstração requerido. Neste cenário, surgem métodos de ensino e aprendizagem que buscam ilustrar os conceitos em pauta através da resolução de problemas, despertando a curiosidade no discente e fazendo-o pesquisar formas de resolver o problema em questão. Pensando nisso, foi desenvolvido um sistema de ensaio didático de viga reduzida formada por perfis metálicos e fixada à uma base feita em acrílico, que pode ser submetida à Momento de Torção, entre outros carregamentos. Essa viga possui vinculação engastada-livre e auxilia os alunos no entendimento de conceitos como Torção, Deslocamento de Rotação, Centro de Torção, e diversos outros, além de possibilitar a medição do ângulo de rotação da viga devido à aplicação de um determinado Momento Torsor. Como resultado obteve-se uma boa compatibilidade entre os conceitos teóricos que serviram de base e o modelo prático, provando ser uma ferramenta de grande utilidade no ensino de Resistência dos Materiais.
\end{abstract}

Palavras-chave: Resistência dos Materiais. Análise de Estruturas. Modelo reduzido. Torção. Ensino da engenharia.

\section{INTRODUÇÃO}

No ensino superior, ao longo dos anos o perfil dos estudantes tem passado por mudanças influenciadas pelo desenvolvimento do mundo em geral, principalmente da tecnologia. Os benefícios são inúmeros, podendo ser destacados a possibilidade da comunicação entre pessoas do mundo todo em questão de segundos e consequentemente troca de informações e conhecimento em tempo real. Porém, essa facilidade desperta na geração de estudantes atual a comodidade, e a faz perder gradativamente a capacidade de imaginar conceitos abstratos ministrados em sala de aula, pois não a exercita com frequência. Junto a isso, com foco no ensino da Resistência dos Materiais e de Análises de Estruturas, há também conceitos básicos no qual se baseiam as metodologias de elaboração de projetos que dificilmente visualiza-se no 
dia a dia, até mesmo na Universidade, o que dificulta seu entendimento e ilustração de como o mesmo ocorre na prática.

Neste cenário a forma mais comum de ensino dos saberes relacionados a essas disciplinas é utilizando metodologias de aprendizagem passiva, onde o professor figura como protagonista do ensino, e o aluno acompanha passivamente a apresentação e explicação dos conteúdos em sala de aula.

Uma abordagem diferente que tem sido cada vez mais aplicadas na engenharia, é o uso de metodologias ativas, em que o protagonismo do aprendizado reside no aluno, tornando o professor um facilitador do conhecimento. Dentre os tipos mais comuns desse método de ensino está o PBL (Problem/Project Based Learning) (Ribeiro 2008; Frezatti et al., 2018; ESCRIVÃO FILHO \& RIBEIRO, 2009).

Uma ferramenta de ensino que vem sendo utilizada com sucesso para auxiliar no aprendizado dos saberes de Resistência dos Materiais e Análise de Estruturas tanto em metodologias passivas, mas principalmente em metodologias ativas é o uso de modelos didáticos, projetados para ilustrar os conceitos e permitir a visualização e verificação de comportamentos e resultados.

Dentro do curso de Engenharia Civil da Universidade Estadual de Londrina (UEL), desde 2013 vêm sendo desenvolvidos modelos reduzidos didáticos quantitativos para o ensino de Resistência dos Materiais e Análise de Estruturas. Dentro os modelos já desenvolvidos que estão sendo aplicados no ensino do Curso vale destacar um modelo de Treliça plana (Campos et al., 2016), de Flambagem (Carbonari et al., 2018) e de vigas isostáticas e hiperestáticas (Campos et al., 2019), dentre outros.

Dentre os diversos saberes da Resistência dos Materiais, o estudo da torção, sobretudo em perfis de parede fina, representa um desafio no ensino, uma vez que os esforços, tensões e o comportamento do centro de torção não são facilmente entendidos pelos alunos apenas com deduções e ilustrações. Falta algo físico palpável que facilite esse processo.

Dentro desta linha, o presente trabalho tem por objetivo a apresentação e aplicação no ensino de um sistema de ensaio didático de perfis de parede fina a torção que permite: Facilitar o entendimento e determinação do centro de torção; Permitir a comparação do comportamento a torção de diferentes perfis de parede fina; aplicar de forma controlada um momento de torção e medir o ângulo de rotação; e ilustrar diferentes aplicações no ensino dos saberes relacionados ao comportamento de barras sobre o efeito de esforço de torção.

\section{CONCEPÇÃO DO SISTEMA}

O modelo foi idealizado na Universidade Estadual de Londrina e executado por uma empresa especializada, e consiste em um sistema de ensaio de perfis de parede fina engastados livre. Na extremidade livre da barra é possível aplicar um momento torsor e medir o ângulo de rotação da seção. Na figura 1 é possível observar o modelo composto por:

- Um suporte em acrílico com um dispositivo que permite engastar diferentes seções transversais, com diferentes tamanhos;

- Um perfil tipo U enrijecido de aço galvanizado (com 0,42 $\mathrm{mm}$ de espessura e 500 mm de comprimento) montado no modelo (a modo de exemplo, pois o modelo é compatível com diversos tipos de perfis);

- Um suporte ajustável (na extremidade livre da barra) que permite a localização do centro de torção, aplicação do momento torção, e fixação da agulha para medição do ângulo de rotação;

- Uma escala que permite a medição da rotação da seção transversal. 
Figura 1 - Sistema de ensaio didático de vigas a torsão.

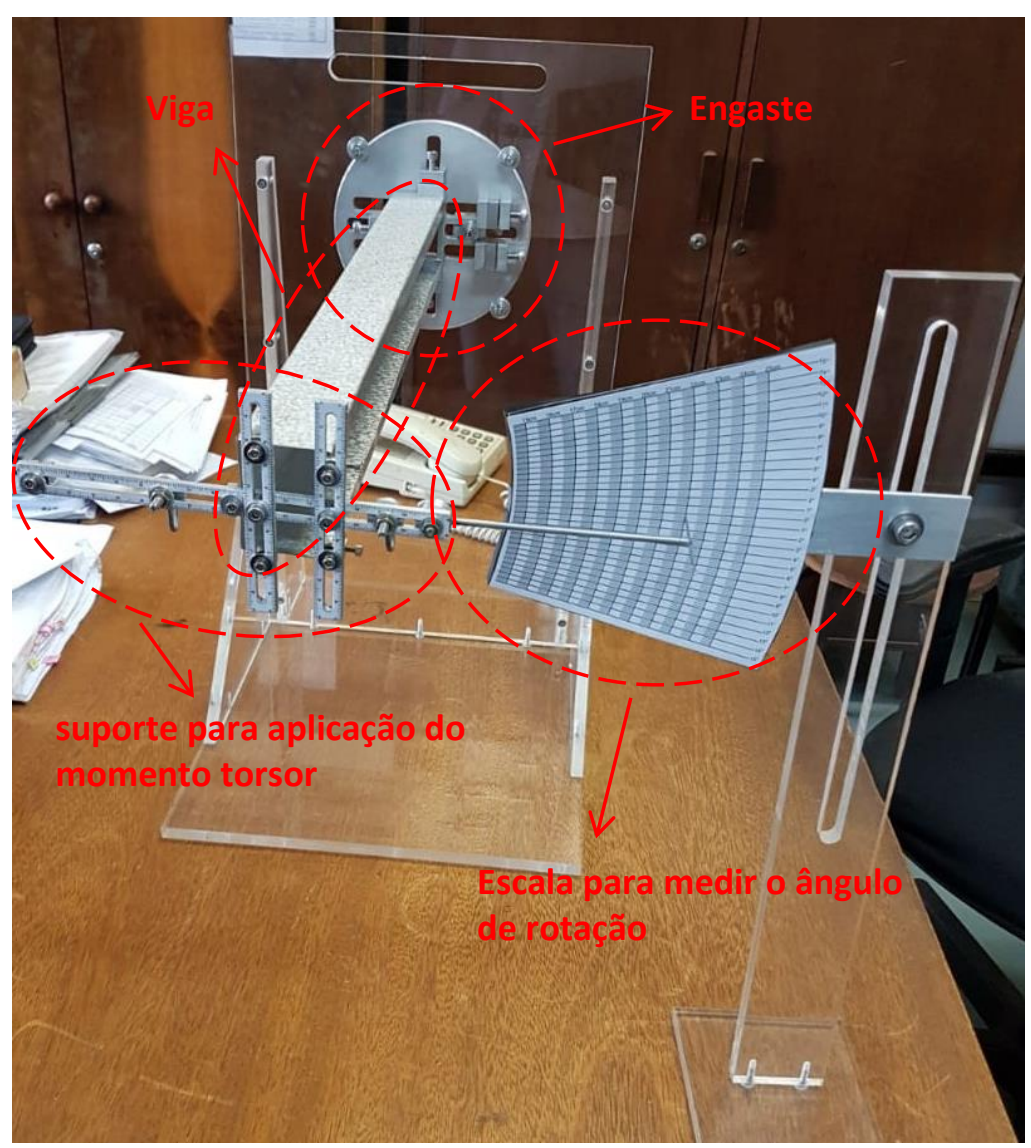

Fonte: Os próprios autores.

\subsection{Vinculação}

A vinculação foi projetada e executada de forma a simular um apoio do terceiro gênero (engaste) próximo do ideal. Na figura 2 é possível observar que a mesma é formada por uma base metálica circular, que permite o posicionamento da seção com diferentes ângulos, fixada na estrutura acrílica. Nesta base metálica há seis peças móveis, que permitem fixar em diferentes posições as bordas do perfil metálico. Esta peça foi concebida para permitir o engasgamento dos perfis ilustrados na figura 3 , entre outros com dimensões diferentes. 


\section{COBENGE C. COBENCE 2020

\section{"Os desafios para formar hoje o engenheiro do amanhã"}

Travamento das mesas e da alma do perfil $U$ enrigecido
Figura 2 - Sistema de ensaio didático de vigas a torsão.

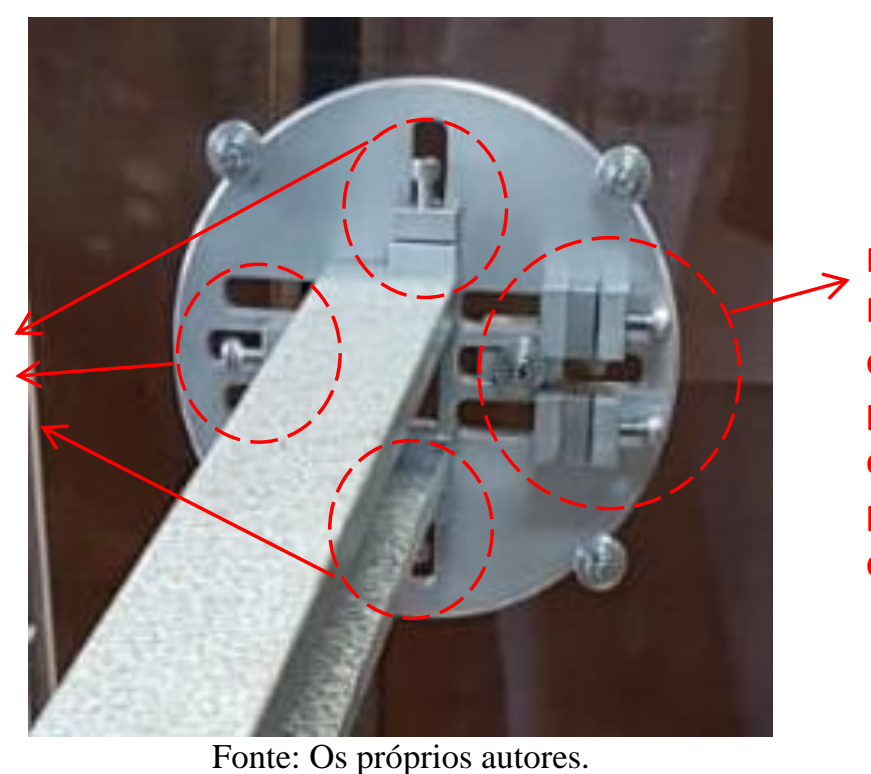

Peças de travamento: Podem ser fixadas em qualquer ponto do trilho, permitindo a fixação de diferentes tamanhos de perfis, com chapas de diferentes espessuras

As chapas metálicas possuem um módulo de elasticidade (E) estimado de $210 \mathrm{GPa}$, comprimento de $500 \mathrm{~mm}$ espessura de $0,42 \mathrm{~mm}$ e demais dimensões ilustradas na Figura 3. Para o desenvolvimento do presente artigo foi utilizado os perfis $U$ e $U$ enrijecido, $\mathrm{Z}, \mathrm{L}$ (cantoneira), H e retangular de seção fechada e aberta. Vale lembrar que é possível fixar outros perfis com diferentes geometrias e espessuras de chapa. A rigidez à torção $\left(\mathrm{GI}_{\mathrm{t}}\right)$ foi calculada analiticamente através de equações da Resistência dos Materiais e um centro de torção (CT), também encontrado através de equações e que influenciará diretamente na intensidade do binário aplicado. Os valores encontrados podem ser vistos no Quadro 1, e as equações utilizadas, além de outras informações sobre o assunto, podem ser encontradas nos seguintes livros: Resistência dos Materiais (HIBBELER, 2009) e Mecânica dos Materiais (BEER et al., 2011).

Quadro 1 - Características geométricas dos perfis.

\begin{tabular}{|c|c|c|}
\hline Perfil & $\begin{array}{c}\text { Inércia à torção } \\
\left(\mathrm{mm}^{4}\right)\end{array}$ & $\begin{array}{c}\text { Distância entre o CT e o baricentro } \\
(\mathrm{mm})\end{array}$ \\
\hline $\mathrm{C}$ & 3,28 & 28,69 \\
\hline C enrijecido & 3,80 & 37,62 \\
\hline $\mathrm{Z}$ & 2,00 & 0,00 \\
\hline $\mathrm{T}$ & 8,95 & 0,00 \\
\hline O aberto & 4,54 & 58,97 \\
\hline $\begin{array}{l}\text { Observação: Módulo de elasticidade transversal }(\mathrm{G})=80,77 \mathrm{GPa} \text {, considerando Coeficiente } \\
\text { de Poisson (v)=0,30 (aço). }\end{array}$
\end{tabular}


Figura 3 - Dimensões das seções transversais dos perfis metálicos (e=espessura).
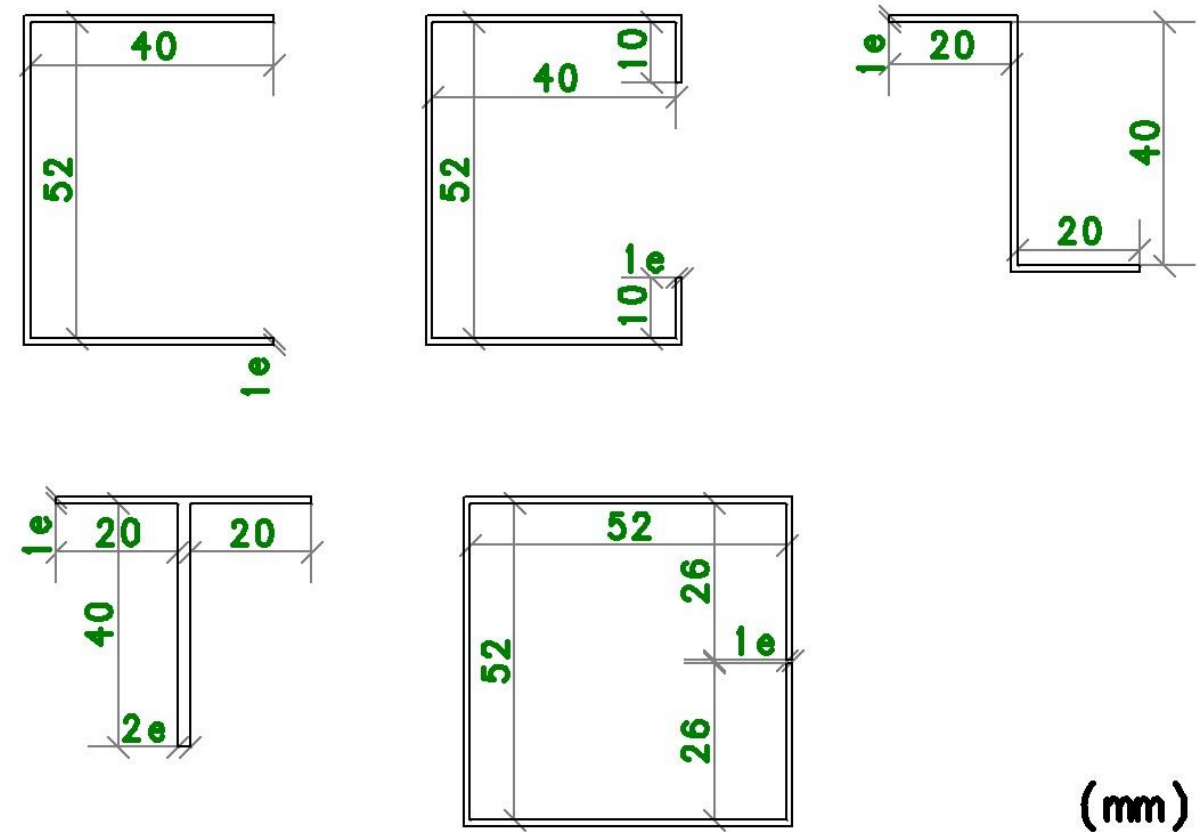

Fonte: Os próprios autores.

\section{$(\mathrm{mm})$}

\subsection{Aplicação do momento torsor e medição da rotação da seção}

A aplicação do momento torsor é feito a partir da aplicação de um binário de forças, igualmente espaçadas do centro de torção, tal como ilustrado na figura 3 . O valor do momento torsor, pela figura será igual a $T=F . d$

Figura 4 - Dimensões das seções transversais dos perfis metálicos.

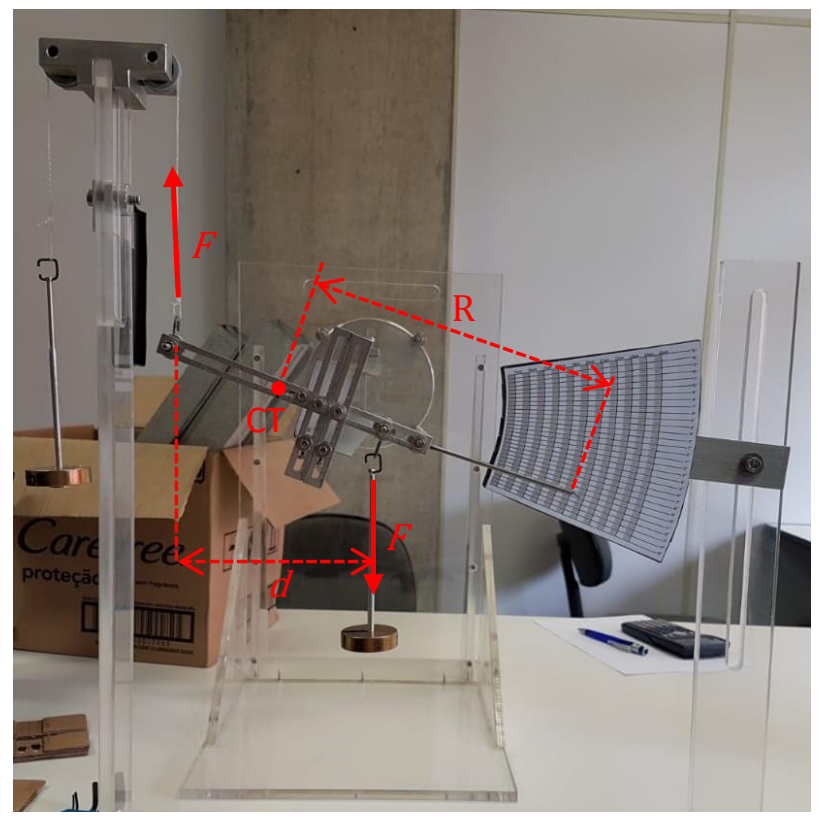

Fonte: Os próprios autores.

Já para a medição da rotação, como cada perfil ensaiado apresenta o CT em uma posição diferentes, o raio (distância da agulha de medição ao centro de torção) será diferente para cada 
perfil ou montagem do ensaio. Para permitir uma medição mais precisa da rotação, a escala de medição apresenta diversas escalas semi-circulares concêntricas, cada uma correspondente a um raio diferente. Assim antes de realizar a medição da rotação deve ser verificada a distância da agulha até o CT (R ilustrada na Figura 3), e posicionar a escala com a agulha sobre a escala semi-circular correspondente a esse raio.

\subsection{Comparação teórico vs experimental}

O Quadro 2 apresenta uma comparação entre os resultados obtidos experimentalmente e os analíticos para diferentes seções transversais, juntamente com suas características geométricas.

Quadro 2 - Comparação dos dados experimentais com os analíticos para diferentes perfis.

\begin{tabular}{|c|c|c|c|c|c|}
\hline Perfil & $\begin{array}{c}\text { Inércia à torção } \\
\left(\mathrm{mm}^{4}\right)\end{array}$ & $\begin{array}{c}\text { Momento aplicado } \\
(\text { Kgf.cm) }\end{array}$ & $\begin{array}{c}\theta \text { analítico } \\
\text { (graus) }\end{array}$ & $\begin{array}{c}\theta \text { experimental } \\
\text { (graus) }\end{array}$ & $\begin{array}{c}\text { Diferença } \\
(\%)\end{array}$ \\
\hline $\mathrm{C}$ & 3,28 & 0,96 & 10,38 & 10,0 & 3,66 \\
\hline C enrijecido & 3,80 & 0,96 & 8,97 & 7,2 & 19,73 \\
\hline $\mathrm{Z}$ & 2,00 & 0,55 & 9,76 & 9,8 & 0,41 \\
\hline $\mathrm{T}$ & 8,95 & 1,08 & 4,28 & 3,0 & 29,90 \\
\hline O aberto & 4,54 & 0,80 & 6,26 & 5,0 & 20,13 \\
\hline
\end{tabular}

Fonte: Os próprios autores.

Como pode ser visto no Quadro 2, em alguns casos as diferenças entre analítico e experimental podem ser maiores do que em outros. Isto pode ser explicado por alguns motivos, como: dificuldade de medir visualmente pequenas variações angulares, onde o critério pode mudar de pessoa para pessoa; facilidade ou dificuldade de manter a viga idealmente engastada após a aplicação de carga, já que alguns perfis necessitam de momentos consideravelmente grandes para fornecerem rotações passíveis de leitura, e isto pode comprometer a vinculação; variação na espessura e/ou nas medidas dos perfis no momento da fabricação; efeito do empenamento desconsiderado no cálculo teórico dos deslocamentos.

\section{APLICAÇÃO NO ENSINO}

A aplicação pode ser feita em sala de aula para o ensino de conceitos básicos das disciplinas de Resistência dos Materiais e Análise de Estruturas seguindo a metodologia de aprendizagem PBL, seguindo uma série de tópicos baseados em ALVES \& ANASTASIOU (2004), que contemplam os processos necessários para se chegar à resolução do problema apresentado. São eles:

- Identificação do problema;

- Obtenção dos dados que o modelo pode fornecer;

- Planejamento, imaginação e elaboração das hipóteses;

- Validação das hipóteses com a prática do modelo;

- Interpretação dos resultados obtidos após a prática.

A identificação do problema se baseia em analisar visualmente o modelo e listar possíveis dados que o modelo pode fornecer, neste caso deslocamento de rotação na 
extremidade livre da viga. Já a obtenção dos dados consiste em coletar todos os dados fornecidos pelo modelo, como as medidas da seção transversal, o comprimento da viga e a forma de vinculação. Planejamento, imaginação e elaboração de hipóteses consistem inicialmente na determinação dos conceitos que envolvem o modelo (Torção e Centro de Torção) e consequentemente as ferramentas (equações para a obtenção do CT das seções transversais, momento de inércia à torção e, com isso, cálculo da rotação) que possibilitarão chegar aos dados listados no primeiro tópico, através de pesquisas por parte dos alunos guiadas pelo docente. Em seguida, organizam-se os dados que serão usados nos cálculos e, se necessário, desprezam-se parcelas que tornarão a análise complexa e/ou inviável, como o efeito de empenamento. A validação das hipóteses consiste na montagem correta do modelo, aplicação do momento de torção, leitura do deslocamento de rotação, análise do comportamento estrutural durante o carregamento e a descarga e finalmente na comparação dos resultados obtidos na prática com os dados analíticos. Por fim, a interpretação dos resultados baseia-se em avaliar todos os dados coletados e calculados, analisar se os mesmo são coerentes com a teoria e se aproximam entre si, listar possíveis razões para as diferenças entre os dois casos e propor uma discussão entre os alunos de novas hipóteses que podem tornar o modelo mais próximo da teoria utilizada inicialmente e formas de calcular as parcelas desprezadas nos cálculos.

Além dos conceitos já abordados anteriormente, como Torção e Centro de Torção o Centro de Cisalhamento (CT), com o modelo é possível também trabalhar outros relacionados às disciplinas de Resistência dos Materiais e Análises de Estruturas, listados no Quadro 2.

Quadro 3 - Outros conceitos possíveis de serem trabalhados através do modelo.

Conceito

\begin{tabular}{|c|c|c|}
\hline \multirow{2}{*}{$\begin{array}{c}\text { Campo de } \\
\text { Validade }\end{array}$} & Lei de Hooke & $\begin{array}{c}\text { Entre os ciclos de carga e descarga, a estrutura retoma sua } \\
\text { geometria original? }\end{array}$ \\
\cline { 2 - 4 } & $\begin{array}{c}\text { Linearidade } \\
\text { geométrica }\end{array}$ & $\begin{array}{c}\text { Os deslocamentos de rotação medidos são proporcionais aos } \\
\text { momentos aplicados e às características geométricas de cada } \\
\text { perfil? }\end{array}$ \\
\hline \multicolumn{2}{|c|}{ Vinculação } & $\begin{array}{r}\text { A partir de qual intensidade do momento o engastamento } \\
\text { completo da viga pode ser comprometido? }\end{array}$ \\
\hline \multirow{2}{*}{$\begin{array}{c}\text { Limitações } \\
\text { teóricas }\end{array}$} & $\begin{array}{c}\text { Momento fletor } \\
\text { e cortante }\end{array}$ & $\begin{array}{c}\text { O sistema adotado para aplicação de Momento Torsor inibe } \\
\text { totalmente o surgimento de Momento Fletor e Cortante? }\end{array}$ \\
\cline { 2 - 4 } & $\begin{array}{c}\text { Empenamento } \\
\text { Até que ponto o efeito do Empenamento pode ser } \\
\text { desconsiderado, sem afetar significativamente a relação entre } \\
\text { a teoria e a hipótese adotada para o modelo? }\end{array}$ \\
\hline \multicolumn{2}{|c|}{ Centro de Torção (CT) } & $\begin{array}{c}\text { Como a seção transversal influencia na posição do Centro de } \\
\text { Torção ou Centro de Cisalhamento (CT)? }\end{array}$ \\
\hline
\end{tabular}

Entre os ciclos de carga e descarga, a estrutura retoma sua geometria original? - A Lei de Hooke, um dos primeiros conceitos abordados por disciplinas que envolvem estruturas em geral, pode ser posta a prova através do modelo proposto, da seguinte forma: aplicam-se cargas na viga de maneira gradativa e acompanha sua deformação, podendo até medir seus deslocamentos de rotação e translação no decorrer do processo e montar um gráfico que relaciona a carga com o deslocamento. Após chegar a certo ponto, realiza-se a retirada das cargas também de forma gradativa e, no final, analisa se a estrutura voltou à sua forma inicial. A linearidade física da viga pode ser validada de duas maneiras: através do gráfico carga 
versus deslocamento, que deve assumir uma relação linear, e através da forma da estrutura após o processo de carga e descarga, que deve voltar à sua posição inicial. Se ambas as condições forem satisfeitas, é válido assumir que o modelo trabalha no regime elástico linear, caso contrário, mede-se os deslocamentos residuais causados pela plastificação do elemento.

Os deslocamentos de rotação medidos são proporcionais aos momentos aplicados e às características geométricas de cada perfil? - Na Resistência dos Materiais, são apresentados os conceitos de Momento de Inércia à Torção $\left(I_{t}\right)$ e mais adiante a relação deste com o ângulo de rotação do elemento gerado pela aplicação de um determinado Momento Torsor, sendo inversamente proporcional. Assim, comparando-se dois perfis com $I_{t}$ 's diferentes, deve apresentar um ângulo de rotação menor aquele com $I_{t}$ maior. Além disso, espera-se ângulos maiores ao passo que o Momento de Torção aplicado também aumenta. Desta forma, demonstra e comprova a aplicação da teoria apresentada em sala de aula.

A partir de qual intensidade do momento o engastamento completo da viga pode ser comprometido? - Na análise de estruturas, são utilizados conceitos de vinculações idealizadas classificadas como primeiro, segundo ou terceiro gênero, de acordo com seu funcionamento. Porém, muitas vezes estas não se assemelham completamente com a realidade. Assim, o modelo permite ao docente demonstrar aos alunos como um engaste funciona na prática, os deslocamentos que o mesmo restringe e como se dá a deformada de uma viga com este tipo de vinculação. Como o engaste do modelo permite vincular diferentes partes do perfil de parede fina, também é possível mostrar a efetividade de uma vinculação da alma, ou das mesas, ou de ambas em conjunto.

O sistema adotado para aplicação de Momento Torsor inibe totalmente o surgimento de Momento Fletor e Cortante? - Conceitos muito estudados nas disciplinas estruturais do curso de Engenharia Civil, as ações do Momento Fletor e da Força Cortante acontecem de formas distintas à ação do Momento de Torção, além de serem mais comuns e familiares aos alunos. O modelo permite ao professor demonstrar visualmente como ocorre cada uma destas ações, fazendo com que o aluno consiga idealizá-las mentalmente em outras ocasiões e distingui-las quando necessário. Quanto ao modelo em si, a hipótese inicialmente adotada é de que age na viga apenas Momento Torsor, sendo necessária a adoção do sistema de polias para torná-la possível, o que permite ao docente mostrar na prática o conceito de força resultante, e também de binário.

Até que ponto o efeito do Empenamento pode ser desconsiderado, sem afetar significativamente a relação entre a teoria e a hipótese adotada para o modelo? - O conceito de Empenamento é discutido principalmente nas disciplinas que abordam perfis metálicos, que são os elementos estruturais mais sujeitos a este efeito. Sendo o modelo composto por vigas de perfis metálicos, se torna possível para o docente trazer este conceito para os alunos e mostrar como o mesmo ocorre na prática. Embora a teoria básica da Resistência dos Materiais não considere o efeito da torção de empenamento, o modelo pode ajudar a comparar a aproximação dos resultados teóricos dos experimentais.

Como a seção transversal influencia na posição do Centro de Torção ou Centro de Cisalhamento (CT)? - Na disciplina de Resistência dos Materiais, o conceito de Centro de Torção ou Centro de Cisalhamento (CT) é muito abstrato e, em alguns casos, difíceis de serem imaginados, além de muitas vezes ser explicado apenas de forma teórica, o que dificulta ainda mais o entendimento do aluno. $\mathrm{O}$ modelo proposto possibilita ao professor discutir algumas propriedades deste conceito em sala de aula, mostrar aos alunos sua influência na prática (principalmente no perfil "C", em que o CT se encontra num ponto fora da seção transversal) e ilustrar a teoria que o envolve. 


\section{CONSIDERAÇÕES FINAIS}

Foi desenvolvido e executado um sistema de ensaio didático e reduzido, qualitativo e quantitativo, de uma viga baseada em perfis metálicos engastada livre que pode ser submetida à ação de Momento Torsor, permitindo a medida de deslocamentos de rotação em sua extremidade livre através de uma agulha metálica e uma escala angular devidamente calibrados.

O modelo em questão apresenta um grande potencial enquanto elemento facilitador no processo de ensino e aprendizagem baseado na PBL (Aprendizagem Baseada em Problemas), possibilitando aos alunos a assimilação de conceitos e métodos de cálculo aplicados na Área Estrutural da Engenharia Civil e visualização dos mesmos na prática.

O modelo, na maior parte dos casos, apresentou pouca variação entre os valores teóricos e experimentais, sendo de $3,66 \%$ para o perfil "C", $0,41 \%$ para o perfil " $Z$ " e $20,13 \%$ para o perfil "O" aberto, atestando que é possível a comparação entre estruturas reais em escala reduzida e a teoria estudada na disciplina de Resistência dos Materiais e Análise de Estruturas

Conclui-se, portanto, que este projeto pode servir como instrumento auxiliador no processo de ensino aprendizagem da Engenharia, assim como na assimilação por parte dos alunos do comportamento do elemento estrutural na prática.

\section{Agradecimentos}

Ao Curso de Especialização em Engenharia de Estruturas do Departamento de Estruturas/CTU/UEL pelo apoio financeiro na execução do modelo. À Prograd/UEL pela formalização do projeto de pesquisa em ensino.

\section{REFERÊNCIAS}

ALVES, Leonir Passate; ANASTASIOU, Léa das Graças Camargos. Estratégias de ensinagem. In: . Processos de Ensinagem na Universidade: pressupostos para as estratégias de trabalho em aula. $3^{\text {a }}$ ed. Joinville: Editora Univille, 2004. p. 68-99.

BASTOS, C. C. Metodologias Ativas. 2006. Disponível em: http://educacaoemedicina.blogspot.com/2006/02/metodologias-ativas.html. Acesso em: 29 abr. 2020.

BEER, Ferdinand Pierre et al. Mecânica dos Materiais. $5^{\text {a }}$ ed. Porto Alegre: AMGH Editora, 2011.

ESCRIVÃO FILHO, E. ; RIBEIRO, L. R. C. Aprendendo com PBL - Aprendizagem Baseada em Problema: relato de uma experiência em cursos de engenharia da EESC-USP. Revista Minerva, São Carlos, v.6, n.1, p. 23-30, 2009.

HIBBELER, Russel Charles. Resistência dos Materiais. $7^{\text {a }}$ ed. São Paulo: Pearson Education, 2009. 


\title{
DEVELOPMENT OF A REDUCED DIDACTIC SYSTEM FOR TESTING THIN-WALLED PROFILES UNDER TORSION TO TEACH MATERIALS STRENGTH
}

\begin{abstract}
In the teaching of Materials Strength and Structural Analysis, one of the main difficulties encountered by teachers is the difficulty for students to understand basic concepts in a more practical way, due mainly to the high degree of abstraction required. In this scenario, teaching and learning methods emerge that seek to illustrate these concepts by solving problems, arousing the curiosity of the students and making them research ways to solve the problems. With that in mind, a didactic test system was developed with a reduced beam formed by metal profiles and fixed to a base made of acrylic. This system can be submitted to Torsion Moment, among other loads. This model of a cantilever beam helps students to understand concepts such as Torsion, Rotation Displacement, Torsion Center, and several others. It also makes it possible to measure the rotation angle of the beam due to the application of a given Torsor Moment. As a result, a good correlation was obtained between the theoretical concepts and the practical model, proving to be a very useful tool in the teaching of Materials Strength.
\end{abstract}

Keywords: Materials Strength. Structural Analysis. Reduced model. Torsion. Engineering teaching. 